\title{
Baseline Models for Bridge Performance Monitoring
}

\author{
Maria Q. Feng ${ }^{1}$; Doo Kie Kim²; Jin-Hak Yi ${ }^{3}$; and Yangbo Chen ${ }^{4}$
}

\begin{abstract}
A baseline model is essential for long-term structural performance monitoring and evaluation. This study represents the first effort in applying a neural network-based system identification technique to establish and update a baseline finite element model of an instrumented highway bridge based on the measurement of its traffic-induced vibrations. The neural network approach is particularly effective in dealing with measurement of a large-scale structure by a limited number of sensors. In this study, sensor systems were installed on two highway bridges and extensive vibration data were collected, based on which modal parameters including natural frequencies and mode shapes of the bridges were extracted using the frequency domain decomposition method as well as the conventional peak picking method. Then an innovative neural network is designed with the input being the modal parameters and the output being the structural parameters of a three-dimensional finite element model of the bridge such as the mass and stiffness elements. After extensively training and testing through finite element analysis, the neural network became capable to identify, with a high level of accuracy, the structural parameter values based on the measured modal parameters, and thus the finite element model of the bridge was successfully updated to a baseline. The neural network developed in this study can be used for future baseline updates as the bridge being monitored periodically over its lifetime.
\end{abstract}

DOI: 10.1061/(ASCE)0733-9399(2004)130:5(562)

CE Database subject headings: Instrumentation; Vibration measurement; Neural networks; Bridges; Performance evaluation; Monitoring; Signal processing.

\section{Introduction}

As the state of the art in bridge design is advancing toward the performance-based design, it becomes increasingly important to monitor and evaluate the long-term structural performance of bridges. Such information is essential in developing new performance criteria for design. Meanwhile, long-term monitoring can produce valuable information and knowledge regarding the structural health condition, which can potentially minimize maintenance cost and enhance longevity of highway bridges (e.g., Aktan et al. 2000). While there are many techniques involved in the structural performance monitoring, they can be broadly categorized as a local or global approach. The former is to obtain information about a relatively small region of a structure by utilizing local nondestructive evaluation (NDE), while the latter uses measurements from a dispersed set of sensors to obtain global infor-

\footnotetext{
${ }^{1}$ Member, Professor, Dept. of Civil and Environmental Engineering, Univ. of California, Irvine, CA 92697-2175. E-mail: mfeng@uci.edu

${ }^{2}$ Assistant Professor, Kunsan National Univ., Kunsan 573-701, Korea; formerly, Visiting Post-Doctoral Researcher, Univ. of California, Irvine, CA 92697-2175. E-mail: kim2kie@kunsan.ac.kr

${ }^{3}$ Research Assistant Professor, Smart Infra-Structure Technology Center, Korea Advanced Institute of Science and Technology, Daejeon 305701, Korea; formerly, Visiting Post-Doctoral Researcher, Univ. of California, Irvine, CA 92697-2175. E-mail: yijh@kaist.ac.kr

${ }^{4}$ Graduate Student, Dept. of Civil and Environmental Engineering, Univ. of California, Irvine, CA 92697-2175. E-mail: yangboc@uci.edu

Note. Associate Editor: Eric N. Landis. Discussion open until October 1, 2004. Separate discussions must be submitted for individual papers. To extend the closing date by one month, a written request must be filed with the ASCE Managing Editor. The manuscript for this paper was submitted for review and possible publication on September 12, 2002; approved on October 4, 2003. This paper is part of the Journal of Engineering Mechanics, Vol. 130, No. 5, May 1, 2004. (CASCE, ISSN 0733-9399/ 2004/5-562-569/\$18.00
}

mation about the condition of the structural system. The global structural performance monitoring involves: (1) Instrumentation of a structure with sensors, and more importantly, (2) methodologies for interpreting the measured data to obtain meaningful information and knowledge concerning the structural performance and integrity. Numerous studies have focused on methodologies based on vibration tests. Structural deterioration or damage causes stiffness degradation that can be reflected, to certain extent, in the changes of structural dynamic characteristics such as frequencies, mode shapes, and mode shape curvatures. Extensive review is given in the references e.g., Salawu (1997) and Doebling et al. (1998).

The writers recently installed sensor systems including accelerometers, pressure sensors, displacement sensors, and embedded strain sensors, on two new highway bridges in Orange County, Calif. Taking advantage of these permanently instrumented bridges, a methodology is being developed for long-term structural performance/integrity evaluation. Integrating the global and local monitoring approaches, this methodology involves the following steps: (1) Developing a three-dimensional (3D) baseline finite element model of the bridge through ambient and/or forced vibration measurements carried out preferably at the very beginning of the bridge life, with a database containing the element stiffness values corresponding to different weather and traffic conditions; (2) periodically updating the baseline model by performing vibration tests; (3) carrying out vibration measurement after a major earthquake or vehicle overloading to globally assess the structural integrity by identifying the change in the baseline model, particularly in the element stiffness; and (4) executing visual and NDE inspection (e.g., Feng et al. 2002) at the identified damage location for more accurate local damage assessment.

In this methodology, a reliable 3D baseline model is essential for successful assessment of the structural performance/integrity. This paper focuses on the development of the baseline model 


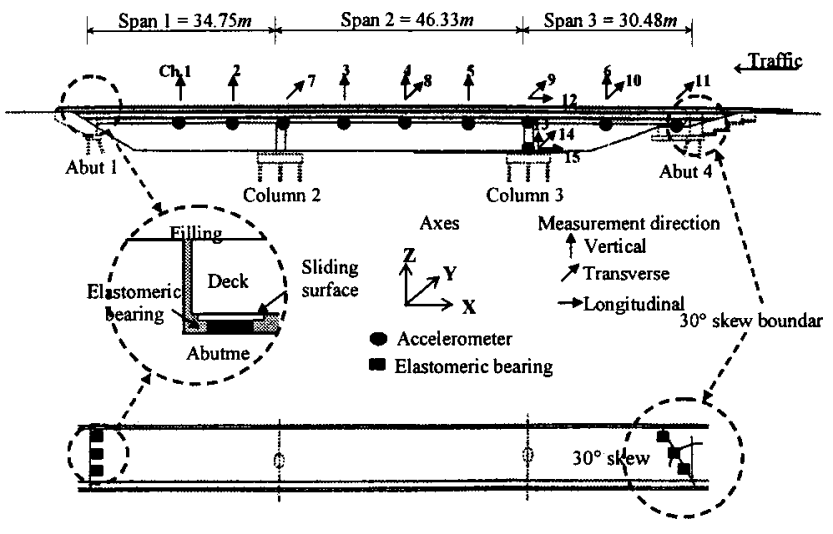

Fig. 1. Jamboree Road overcrossing and accelerometer arrangement

using neural network-based system identification technique and vibration data measured at the instrumented bridges. To be more specific, instrumentation of the two new highway bridges with sensors is first presented, then vibration testing and extraction of bridge dynamic characteristics (modal parameters) using different techniques are discussed, and finally the neural network-based system identification technique is presented to develop a 3D baseline model for one of the instrumented bridges based on its measured dynamic characteristics.

\section{Instrumentation of Two Bridges}

In order to study long-term structural performance and integrity of highway bridges, the writers recently instrumented two new highway bridges; the Jamboree Road overcrossing and the West Street on-ramp.

\section{Description of Bridges}

The Jamboree Road overcrossing, as shown in Fig. 1, is a typical three-span continuous cast-in-place prestressed post-tension boxgirder bridge of the Eastern Transportation Corridor, located in Irvine, Calif. The total length of the bridge is $110.9 \mathrm{~m}$ with each span length of $35.5,46.1$, and $30.3 \mathrm{~m}$. The bridge is supported on two monolithic single columns and sliding bearings on both abutments. The sliding bearings allow creep, shrinkage, and thermal expansion or contraction.

The West Street on-ramp, as shown in Fig. 2, is a three-span

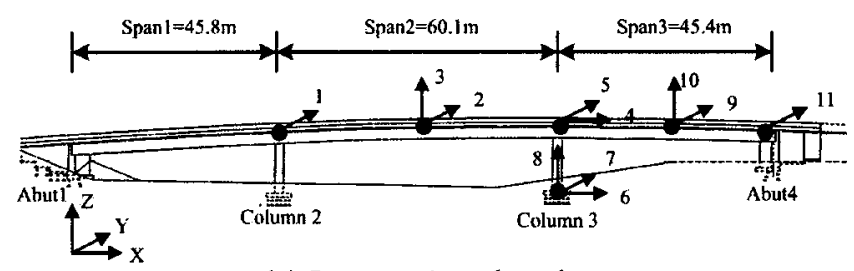

(a) Layout Acceleration

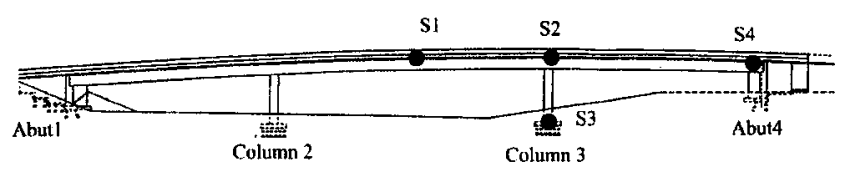

(b) Layout of strain gauges

Fig. 2. West Street on-ramp continuous and curved cast-in-place prestressed post-tension boxgirder bridge. The total length of the bridge is $151.3 \mathrm{~m}$ with the span lengths of $45.8,60.1$, and $45.4 \mathrm{~m}$. The bridge is supported by two fixed columns and sliding bearings on both abutments similar to the Jamboree Road overcrossing. This bridge is highly curved and inclined with a $12 \%$ superelevation.

These two bridges represent two types of typical highway bridges in Calififornia: One is an over- or undercrossing which is usually straight, and the other is an on-ramp which is curved in plane.

\section{Monitoring System}

Monitoring systems involving not only accelerometers but also strain gages were installed at both super- and substructures. Monitoring vibration of the substructures is also of importance since the columns turned out to be one of the most vulnerable structural components of highway bridges under damaging earthquakes. Uniaxial, biaxial, and triaxial force-balance servo-type accelerometers were permanently installed on both of the bridges. The locations of the accelerometers at Jamboree Road overcrossing are shown in Fig. 1, and they are placed along the center line of the bottom of the girder to minimize the torsional effect of the bridge box girder. Strain gages, which are microdisplacement sensors, were permanently embedded in concrete members of the West Street on-ramp to measure dynamic strains. In order to measure the movement of the superstructure with respect to the abutment due to shortening, creep, shrinkage, as well as seismic excitations, a displacement sensor was installed at an abutment for each bridge. To measure the soil pressures at the abutment during earthquakes, a soil pressure sensor was installed on the back wall of the abutment at the West Street on-ramp. Uninterruptible power supply systems were installed at the bridges to prevent power failure due to unexpected events such as earthquakes. In the Jamboree Road overcrossing, a solar power supply system was also installed as a backup to power the monitoring system.

\section{Vibration Tests}

With the sensors installed on the bridges, traffic-induced vibrations were measured, based on which the dynamic characteristics (modal parameters) were extracted using the signal processing techniques discussed as follows.

\section{Signal Processing Techniques}

For traffic-induced vibration, the input loads are normally difficult to measure, and thus the dynamic characteristics are estimated based on the responses only. The peak picking (PP) (Bendat and Piersol 1993; Ewins 1999) and the frequency domain decomposition (FDD) (Otte et al. 1990; Brinker et al. 2001) methods were used to derive modal parameters from the vibration measurements without requiring information about input loads.

The PP method widely used in practice gives reasonable estimates of natural frequencies and mode shapes if the vibration modes are well separated. The FDD method may be used to separate close modes, thus obtaining better estimates. In the FDD technique, taking the singular value decomposition, the spectral density matrix $\mathbf{S}_{\mathbf{Y Y}}(\omega)$ of the response vector $\mathbf{Y}(t)$ is decomposed into

$$
\mathbf{S}_{\mathbf{Y Y}}(\omega)=\mathbf{U}(\omega) \mathbf{\Sigma}(\omega) \mathbf{U}^{H}(\omega)
$$

where $\boldsymbol{\Sigma}(\omega)=$ diagonal matrix of the singular values; $\mathbf{U}(\omega)$ = unitary matrix of the singular vectors; and the superscript $H$ 


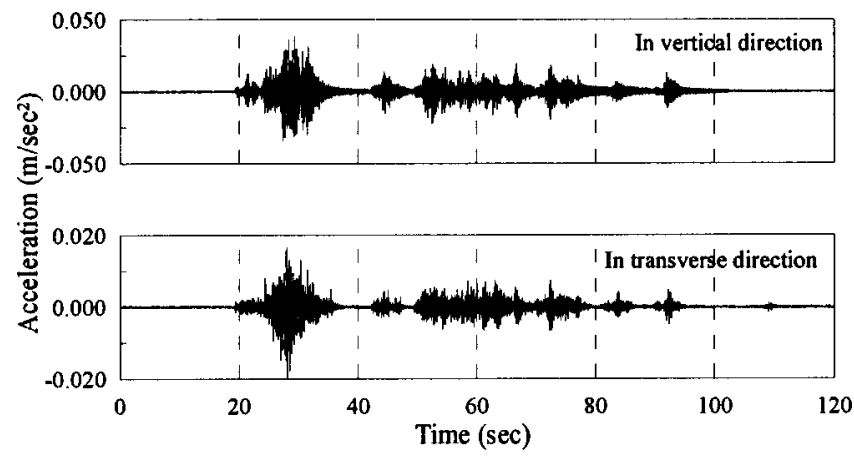

Fig. 3. Typical accelerations in middle of span 2

denotes the complex conjugate and transpose. It has been shown by Brinker et al. (2001) that, when the structure is loaded with broadband excitation, near the natural frequencies of the modes, $\Sigma(\omega)$ contains a set of functions which are approximations of the autospectral density functions of the modes' equivalent single degree-of-freedom (SDOF) systems in the normal coordinates, while the vectors in $\mathbf{U}(\omega)$ hold the mode shapes of the corresponding modes. The approximation becomes exact in the case where the loading is white noise, the structure is lightly damped, and the mode shapes of the close modes are geometrically orthogonal. If these assumptions are not satisfied, the decomposition into SDOF systems is still expected to be more accurate than those of the classical approaches including PP.

The random decrement (RD) technique, sometimes referred to as randomdec, was used to average the measured data in order to reduce the noises (Yang et al. 1985; Asmussen and Brinker 1996). The fundamental concept of the RD technique is based on the fact that the response of a structure under random excitation is composed of a deterministic part (associated with the initial conditions) and a random part (associated with the random excitation). By averaging sufficient sample responses, the random part associated with the zero mean random excitation will average out, leaving only the deterministic part. The RD signature vector $\mathbf{z}(\tau, x)$ can be obtained as

$$
\mathbf{z}(\tau, x)=\frac{1}{N} \sum_{i=1}^{N} \mathbf{y}\left(\tau+t_{i}, x\right)
$$

where $\mathbf{y}(t, x)=$ measurement at $x ; t_{i} \mathrm{~s}=$ time instances satisfying a prescribed triggering condition at a leading station; $N=$ number of triggering points; and $\tau=$ time variable. Therefore, the RD signature will exhibit the free vibration decay associated with the initial condition same to the prescribed triggering condition. In this study, the PP technique is then apply to the RD signature in fre-

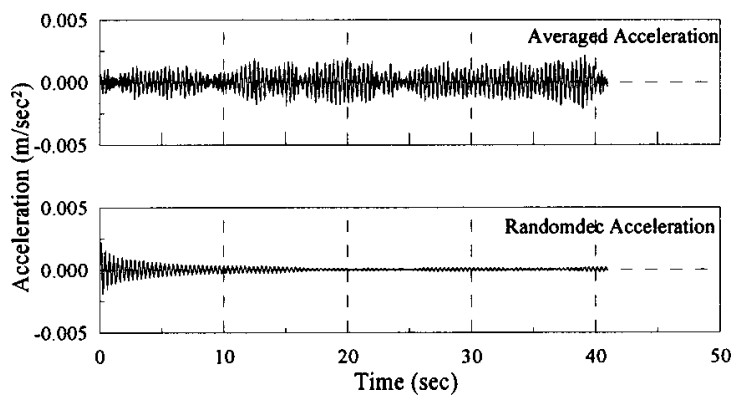

Fig. 4. Processed accelerations in middle of span 2

quency domain to obtain the modal parameters. The combination of PP and RD is hereafter referred to as the PP/RD method.

\section{Ambient Vibration Tests}

Ambient vibration tests were carried out for the Jamboree Road overcrossing. The accelerations at the various locations on the box-girder and a column (shown in Fig. 1) were measured with a sampling frequency of $100 \mathrm{~Hz}$, with the corresponding Nyquist frequency being $50 \mathrm{~Hz}$, which is sufficient for identifying the major lower modes of the bridges. Typical time histories of accelerations measured in the middle of Span 2 in the vertical and in the transverse directions are shown in Fig. 3. The amplitudes of accelerations in the transverse direction are approximately half of those in the vertical direction, as the traffic loads mainly induce vibrations in the vertical direction.

In total, 82 sets of data measured in the daytime were processed, in which the time length of each data set is $10 \mathrm{~min}$. The PP/RD method and the FDD method were used to extract modal parameters. The RD signature in the PP/RD method was obtained with a prescribed initial condition of positive zero crossing. In the FDD method, the spectral density matrix $\mathbf{S}_{\mathbf{Y Y}}(\omega)$ was obtained from the averaged vibration data with $25 \%$ overlapping. Fig. 4 shows the time histories of the RD signature and the averaged acceleration in the middle of Span 2. A $0.5 \mathrm{~Hz}$ high-pass filter was used to reduce bias errors, and extensive averaging contributed to reducing random errors.

The natural frequencies of the bridge derived using the two different methods are shown together in Table 1, in which the in-plane modes are the modes in the vertical and longitudinal directions, while the out-of-plane modes are the modes in the transverse direction. The table also shows the natural frequencies calculated from a preliminary finite element (FE) analysis using a model based on the bridge's design drawings (to be described later). There are not much differences between the frequencies

Table 1. Comparison of Natural Frequencies of Jamboree Road Overcrossing (Hz)

\begin{tabular}{|c|c|c|c|c|c|c|c|c|}
\hline \multirow[b]{3}{*}{ Mode number } & \multicolumn{4}{|c|}{ In-plane mode } & \multicolumn{4}{|c|}{ Out-of-plane mode } \\
\hline & \multicolumn{2}{|c|}{ Measured } & \multicolumn{2}{|c|}{ Numerical } & \multicolumn{2}{|c|}{ Measured } & \multicolumn{2}{|c|}{ Numerical } \\
\hline & PP/RD & FDD & Preliminary FE & Baseline FE & PP/RD & FDD & Preliminary FE & Baseline FE \\
\hline 1 & 2.954 & 2.954 & $2.889(2.20)$ & $2.947(0.24)$ & 2.612 & 2.612 & $3.126(19.7)$ & $2.633(0.80)$ \\
\hline 2 & 4.077 & 3.979 & $3.716(6.61)$ & $3.955(0.60)$ & 5.004 & 5.004 & $4.535(9.37)$ & $4.982(0.44)$ \\
\hline 3 & 4.638 & 4.638 & $4.654(0.35)$ & $4.798(3.45)$ & 9.940 & 9.936 & $10.509(5.77)$ & $9.745(1.92)$ \\
\hline 4 & 6.323 & 6.250 & $5.721(8.46)$ & $5.831(6.70)$ & - & - & - & - \\
\hline 5 & 8.813 & 8.911 & $9.400(5.49)$ & $9.565(7.34)$ & - & - & - & - \\
\hline
\end{tabular}

Note: $\mathrm{PP}=$ peak picking; $\mathrm{RD}=$ random decrement; $\mathrm{FDD}=$ frequency domain decomposition; and $\mathrm{FE}=$ finite element. The values in parentheses are the differences $(\%)$ between the numerical and the experimental natural frequencies (obtained from FDD). 


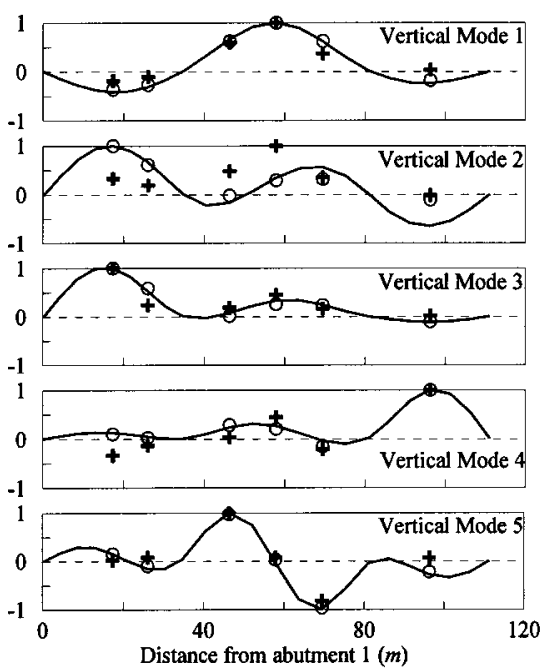

(a) In-plane Modes

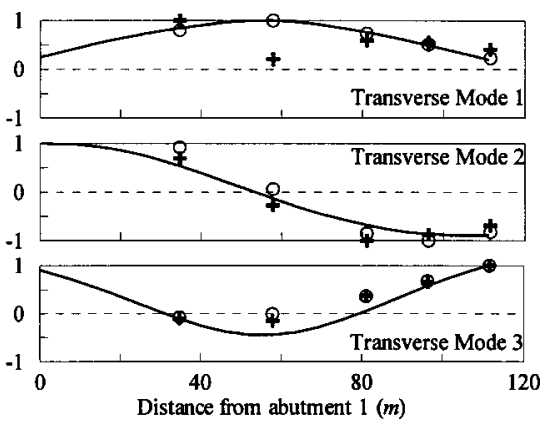

Preliminary FE analysis
PP/RD
FDD

(b) Out-of-plane Modes

Fig. 5. Comparison of mode shapes ( $F E=$ finite element; $P P=$ peak picking; $R D=$ random decrement; and $F D D=$ frequency domain decomposition)

derived from the two methods based on the measured data, but the calculated frequencies do not agree with the measured ones.

Fig. 5 shows the mode shapes corresponding to the natural frequencies. It is observed that the high and the horizontal modes contain more noises than the low and the vertical modes, as traffic loads mainly excite vertical vibration and the frequency contents of the loads are relatively low. The measurement on the top of column 3 in the longitudinal direction was important to differentiate the second and the third vertical modes, because the two modes are very similar in the vertical direction but different in the longitudinal direction. As shown in Fig. 5, the longitudinal displacement of the second vertical mode at column 3 was to the right, whereas that of the third mode was to the left. This is because that the maximum vertical modal displacement of Span 2 is larger than that of Span 3 in the second mode, and vice versa in the third mode.

In Fig. 5, the mode shapes estimated from the PP/RD and FDD methods were also compared with those from the preliminary FE analysis. Observing the mode shapes, those estimated by the FDD method agree better with those from the FE analysis than those estimated by the PP/RD methods. The mode shapes estimated by the FDD method appear more reasonable than those by the PP/RD method.

\section{Forced Vibration Tests}

Braking and bumping vibration tests were carried out using a water truck at the final constructional stages of the West Street on-ramp before it opened to traffic. Braking and bumping forces applied by the water truck at the middle point of each span induced bridge vibration in the longitudinal and vertical directions. The bumping force was applied vertically using a bumper, and the braking force was created by sudden stoppage during traveling. The fully loaded water truck weighed approximately $15,000 \mathrm{~kg}$. Typical time histories of accelerations of the superstructure are shown in Fig. 6. The amplitudes of accelerations in the transverse direction are relatively smaller than those in the vertical direction. More importantly, the amplitudes of the accelerations in the bumping vibration tests were greater than those in the braking vibration tests.
Four sets of data from the braking vibration tests and three sets from the bumping vibration tests were processed, in which the time length of each data set is $10 \mathrm{~min}$ and all the data were collected at night with little traffic in the neighboring freeways and the bridge blocked. The FDD method was used to extract modal parameters from the averaged vibration data with $25 \%$ overlapping. The sampling frequency is $100 \mathrm{~Hz}$ and the time length of the averaged data 40.96 s. Since the vertical and the transverse modes are coupled together due to the curved shape of the bridge, the first two modes were estimated utilizing simultaneously the vertical and transverse vibration signals. The resulting natural frequencies are shown in Table 2 . It is observed that the

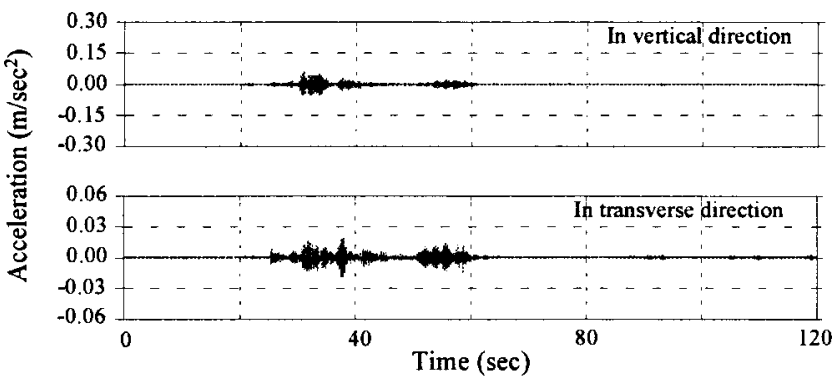

(a) Braking tests

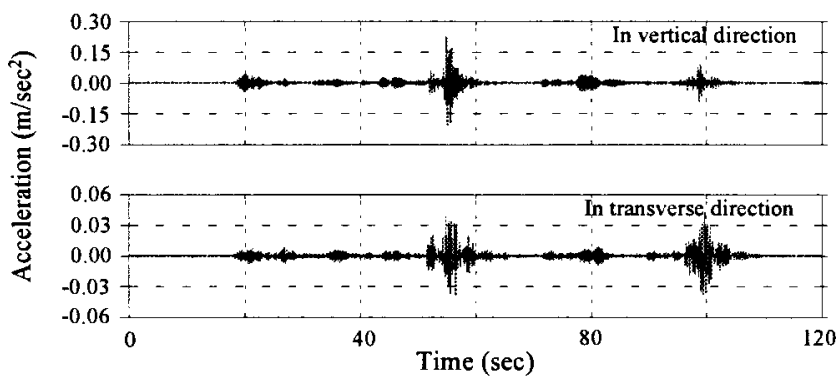

(b) Bumping tests

Fig. 6. Typical acceleration time history by braking and bumping tests 
Table 2. Comparison of Natural Frequencies of West Street On-Ramp (Hz)

\begin{tabular}{lccccc}
\hline Test & \multicolumn{2}{c}{ Braking vibration test } & & \multicolumn{2}{c}{ Bumping vibration test } \\
\cline { 2 - 3 } \cline { 5 - 6 } Mode & Vertical & Transverse & & Vertical & Transverse \\
\hline 1 & 2.148 & 2.148 & & $2.075(3.40)$ & $2.124(1.12)$ \\
2 & 2.465 & 2.465 & & $2.441(0.97)$ & $2.441(0.97)$ \\
\hline
\end{tabular}

Note: The values in the parentheses are the differences $(\%)$ between breaking and bumping results.

natural frequencies estimated from the bumping tests are $3.4 \%$ smaller than those from the braking tests, although these tests were performed with the same water truck and at the same loading locations. The difference in vibration amplitudes caused by the bumping and breaking tests may have contributed to the difference in the natural frequencies. This suggests a need for further study on the nonlinear effect associated with different excitation levels on the modal parameters.

\section{Development of Baseline Model}

A baseline model has been developed using the Jamboree Road overcrossing as an example. A preliminary FE model was first developed based on the bridge design drawing, and then updated to the baseline based on the measured dynamic characteristics by applying a the neural network-based system identification technique.

\section{Preliminary Finite Element Analysis}

A 3D preliminary FE model, as shown in Fig. 7, was developed for the bridge. The super- and substructures were modeled as 3D frame elements by using SAP2000 (1999). The cross-section area and moment of inertia for each element were calculated from the design drawings and listed in Table 3. Usually the abutments are assumed as hinged or fixed supporting conditions. However, recent research reports reveal that they are neither hinged nor fixed and the stiffness affects the behavior of bridges (Sanayei et al. 1999; Yun et al. 2002). In this study, considering that the model is for analyzing bridge response to operational (traffic) loads, the abutment was modeled as linear longitudinal and transverse rotational springs with the stiffness values determined based on the Federal Highway Administration (FHWA) recommendation (FWHA 1996) and listed in Table 3. The natural frequencies and the mode shapes computed using this preliminary FE model are shown in Table 1 and Fig. 5. The difference as shown in the parentheses between the calculated and the measured natural frequencies suggests the need for updating the preliminary FE model using the measurement results.

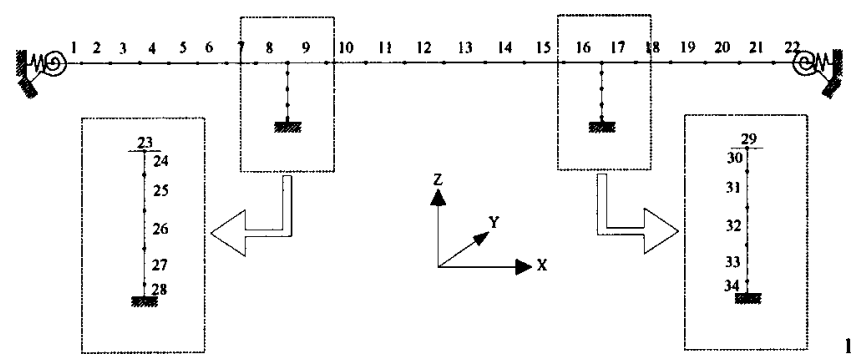

Fig. 7. Finite element model of Jamboree road overcrossing
Table 3. Structural Parameters for Jamboree Road Overcrossing

\begin{tabular}{lccccccc}
\hline & \multicolumn{9}{c}{$\begin{array}{c}\text { Moments of } \\
\text { inertia }\left(\mathrm{m}^{4}\right)\end{array}$} & & \multicolumn{2}{c}{ Spring stiffness } \\
\cline { 3 - 5 } \cline { 8 - 9 } Element & $\begin{array}{c}\text { Area } \\
\left(\mathrm{m}^{2}\right)\end{array}$ & $I_{X}$ & $I_{Y}$ & $I_{Z}$ & & $k_{l 1}^{\text {abut }}, k_{l 4}^{\text {abut }}$ & $k_{r 1}^{\text {abut }}, k_{r 4}^{\text {abut }}$ \\
\hline Deck & 5.94 & 7.63 & 3.01 & 5.94 & & N/A & N/A \\
Column & 3.53 & 2.51 & 0.72 & 1.51 & & N/A & N/A \\
Abutment & N/A & N/A & N/A & N/A & & $1.996 \times 10^{8}$ & $7.582 \times 10^{8}$ \\
\hline
\end{tabular}

Note: Not available.

Sensitivity of the modal parameters to column damage was analyzed. The damage is defined as the decrease of flexural stiffness. Seismic damage often occurs at the plastic hinge zones that are at the top and bottom sections of the columns in the longitudinal direction and at the bottom sections of the columns for the transverse direction considering the maximum bending moments. Fig. 8 shows the column damage decreases the lower natural fre-
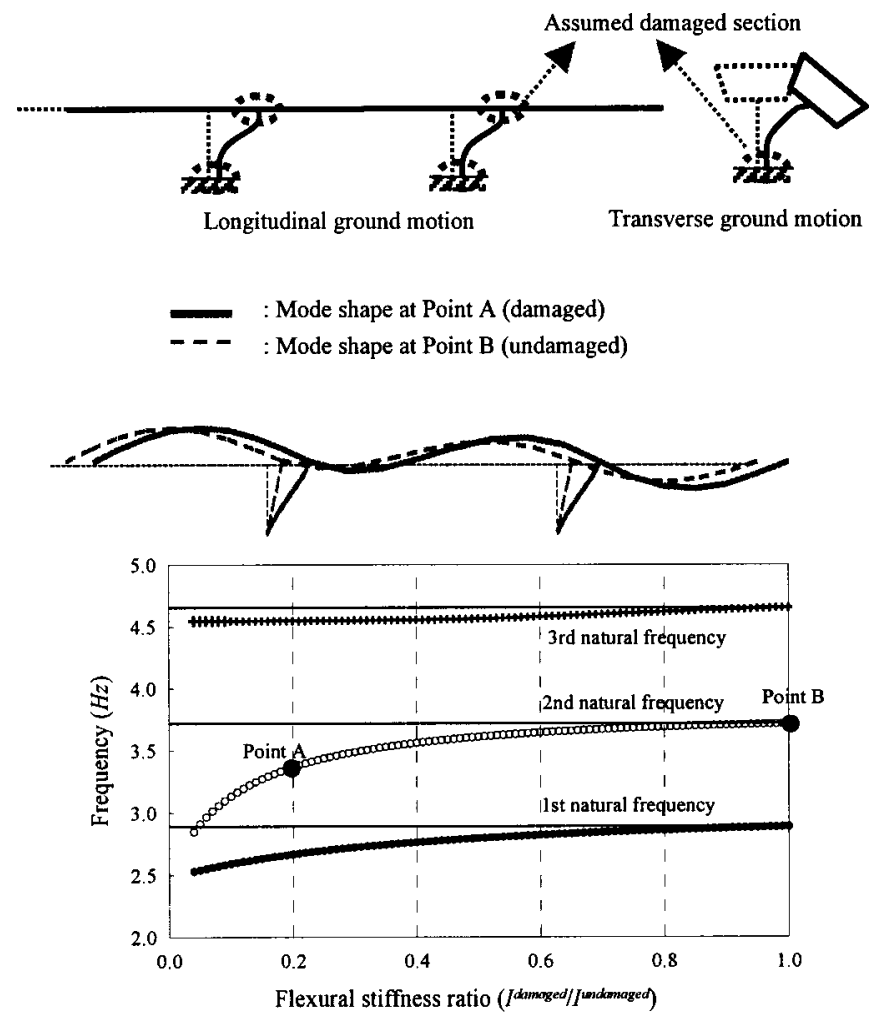

(a) In-plane mode by longitudinal ground motion

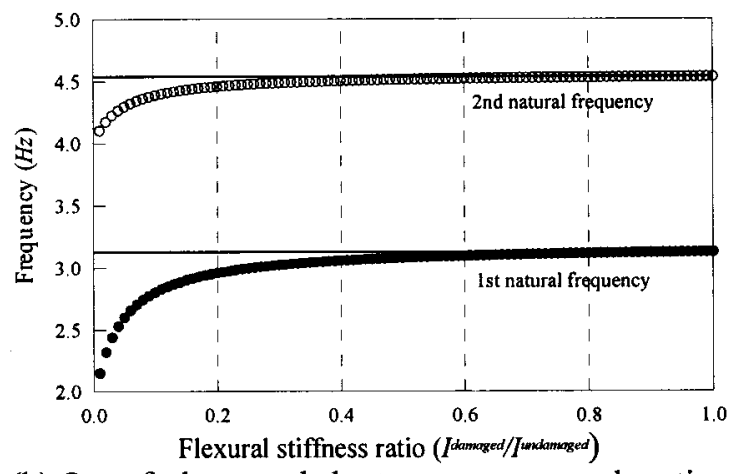

(b) Out-of-plane mode by transverse ground motion

Fig. 8. Sensitivity of natural frequency for column damages 

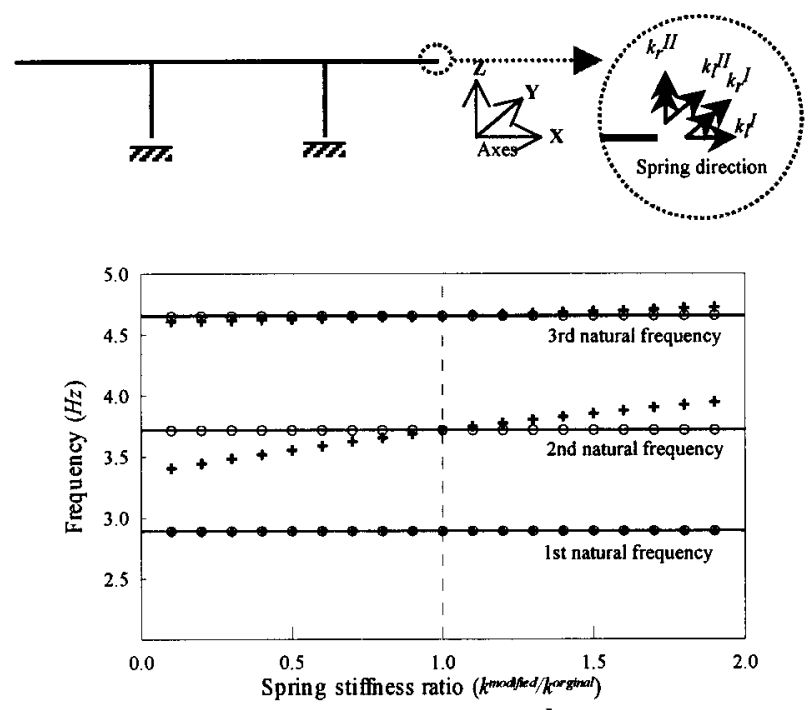

(a) In-plane mode $\left(\boldsymbol{\uparrow}\right.$ : longitudinal $\left(k_{l}^{I}\right) \bigcirc$ : rotational $\left.\left(k_{r}^{l}\right)\right)$

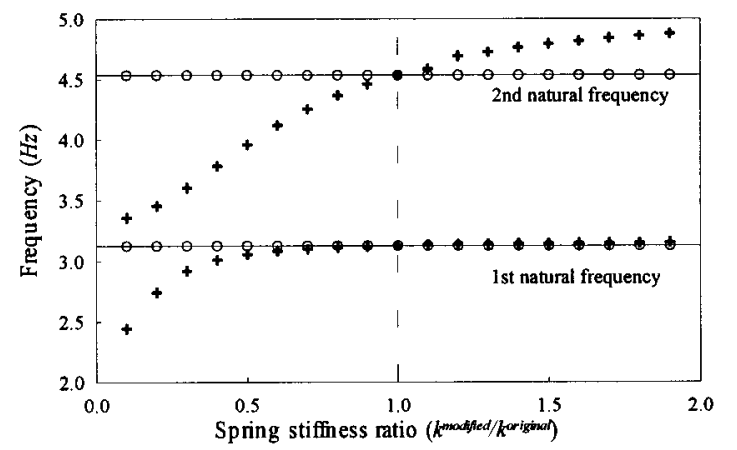

(b) Out-of-plane mode ( $\$$ : transverse $\left(k_{l}^{I I}\right)$ O: rotational

$$
\left.\left(k_{r}^{I I}\right)\right)
$$

Fig. 9. Sensitivity of natural frequency to spring stiffness

quencies and increases the rotations of columns in the mode shape. Therefore, installing accelerometers in the longitudinal and transverse directions at the top of columns is important for seismic damage detection.

Sensitivity analyses of the modal parameters with respect to the abutment spring stiffness were also performed. The spring stiffness at abutment 4 changed from 10 to $190 \%$ of the original value. As shown in Fig. 9, the modal parameters are not sensitive to the rotational stiffness.

\section{Neural Network-Based System Identification}

The back propagation neural network technique (e.g., Feng and Bahng 1999; Masri et al. 2000; Yun et al. 2001) was applied to update the preliminary FE model to the baseline model based on the measured dynamic characteristics of the bridge. The neural network-based system identification method has several advantages compared with conventional system identification methods. The neural network approach is more capable of building a baseline FE model based on the partially and incompletely measured components of the mode shapes due to the limited sensor number, and on only a few lower modes extractable from the vibration signals. Furthermore, it is very convenient to use the neural network to parameterize any properties of the structures, such as the effective shear area, as the unknowns to be identified. In contrast to many system identification methods in which the sensitivity

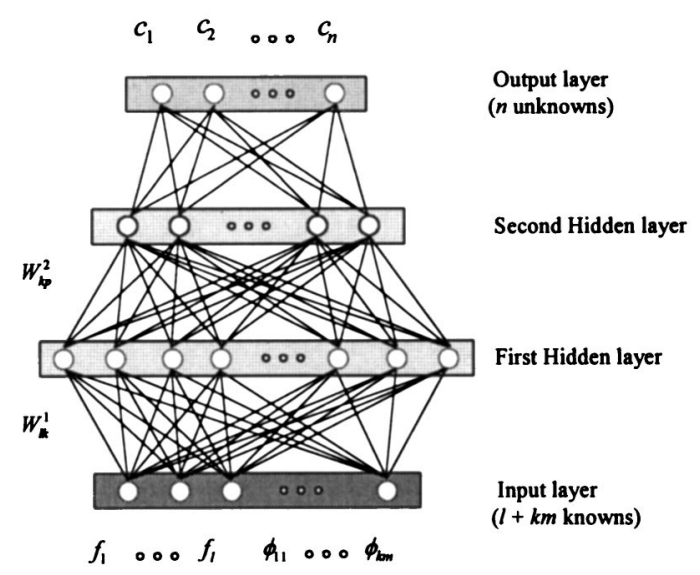

Fig. 10. Back propagation neural network

matrix may become unstable especially for complex structural systems, the neural network approach does not require calculation of the sensitivity matrix, and thus can be applied to the complex civil engineering structures avoiding the numerical difficulty.

As shown in Fig. 10, the neural network consists of an input layer, hidden layers, and an output layer. The relationship between input and output of a neural network can be nonlinear or linear, and its characteristics are determined by the weights assigned to the connections between the neurons in two adjacent layers. Changing these weights will change the input/output relationship of the network. A systematic way of determining the weights of the network to achieve a desired input/output relationship is referred to as a training or learning algorithm. The standard back propagation algorithm was used in this study, for training the neural network to identify structural parameters (the stiffness and the mass matrices of the bridge, and the spring stiffness at the abutments) from measured natural frequencies and mode shapes.

The procedure of the neural network-based identification involves the following steps: (1) Determining the types of input and output patterns; (2) preparing the training and testing patterns through FE analyses; (3) training the neural network using the back propagation algorithm; and finally (4) estimating the structural parameters of the baseline FE model by inputting the measured natural frequencies and mode shapes to the well trained neural network.

In the present study, the input pattern consists of the natural frequencies and the mode shapes

input pattern vector

$$
=\left\{f_{1}, \ldots, f_{i}, \ldots, f_{l},\left[\left(\phi_{1 i}, \ldots, \phi_{j i}, \ldots, \phi_{k i}\right), i=1, \ldots, m\right]\right\}
$$

where $f_{i}=i$ th natural frequency; $\phi_{j i}$ denotes the $j$ th component of the $i$ th mode shape $\boldsymbol{\varphi}_{i}$ which is normalized as $\boldsymbol{\varphi}_{i}^{T} \boldsymbol{\varphi}_{i}=1 ; k$ $=$ number of accelerometers installed on a structure; and $l$ and $m=$ number of natural frequencies and mode shapes considered, respectively. The output pattern consists of correction coefficients of structural parameters

$$
\text { output pattern vector }=\left\{c_{1}, \ldots, c_{n}\right\}
$$

where $c_{1}, \cdots, c_{n}=$ correction coefficients for the FE model's structural parameters (described as a portion of the original values in the preliminary FE model). Training input-output data sets 
Table 4. Neural Network Output for Verification

\begin{tabular}{|c|c|c|c|c|c|c|c|c|c|c|c|}
\hline \multirow{2}{*}{$\begin{array}{l}\text { Correction } \\
\text { coefficient }\end{array}$} & \multicolumn{3}{|c|}{ Target } & \multicolumn{3}{|c|}{$\begin{array}{l}\text { Output with identification } \\
\text { of rotational stiffness }\end{array}$} & \multirow{2}{*}{$\begin{array}{c}\text { Mean sizing } \\
\text { error }^{\mathrm{a}} \\
(\%)\end{array}$} & \multicolumn{3}{|c|}{$\begin{array}{l}\text { Output without identification } \\
\text { of rotational Stiffness }\end{array}$} & \multirow{2}{*}{$\begin{array}{c}\text { Mean sizing } \\
\text { error }^{\mathrm{a}} \\
(\%)\end{array}$} \\
\hline & 1 & 2 & 3 & 1 & 2 & 3 & & 1 & 2 & 3 & \\
\hline \multicolumn{12}{|c|}{ In-plane mode } \\
\hline$c_{1}^{\mathrm{I}}$ & 1.2 & 1.2 & 1.3 & 1.34 & 1.22 & 1.32 & 6.00 & 1.19 & 1.20 & 1.32 & 1.00 \\
\hline$c_{2}^{\mathrm{I}}$ & 0.5 & 1.3 & 1.4 & 0.55 & 1.26 & 1.41 & 3.33 & 0.51 & 1.31 & 1.40 & 0.67 \\
\hline$c_{3}^{\mathrm{I}}$ & 0.7 & 0.7 & 0.5 & 0.76 & 0.71 & 0.57 & 4.67 & 0.70 & 0.70 & 0.50 & 0.00 \\
\hline$c_{4}^{\mathrm{I}}$ & 0.9 & 0.5 & 1.3 & 0.97 & 0.56 & 1.27 & 5.33 & 0.90 & 0.50 & 1.31 & 0.33 \\
\hline$c_{5}^{\mathrm{I}}$ & 1.1 & 1.6 & 1.0 & 1.21 & 1.56 & 0.97 & 6.00 & 1.09 & 1.58 & 1.00 & 1.00 \\
\hline$c_{6}^{\mathrm{I}}$ & 1.5 & 1.4 & 1.5 & 1.38 & 1.05 & 0.97 & 33.33 & - & - & - & - \\
\hline$c_{7}^{\mathrm{I}}$ & 0.1 & 0.6 & 1.1 & 0.15 & 0.72 & 1.12 & 6.33 & 0.15 & 0.56 & 1.12 & 3.67 \\
\hline$c_{8}^{\mathrm{I}}$ & 0.2 & 1.8 & 0.3 & 0.26 & 1.80 & 0.74 & 16.67 & - & - & - & - \\
\hline \multicolumn{12}{|c|}{ Out-of-plane mode } \\
\hline$c_{1}^{\mathrm{II}}$ & 1.2 & 1.2 & 1.3 & 1.16 & 1.11 & 1.35 & 6.00 & 1.20 & 1.20 & 1.30 & 0.00 \\
\hline$c_{2}^{\mathrm{II}}$ & 1.1 & 1.0 & 1.0 & 1.08 & 0.91 & 0.95 & 5.33 & 1.10 & 1.01 & 1.00 & 0.33 \\
\hline$c_{3}^{\mathrm{II}}$ & 0.6 & 0.5 & 0.1 & 0.60 & 0.49 & 0.13 & 1.33 & 0.59 & 0.51 & 0.12 & 1.33 \\
\hline$c_{4}^{\mathrm{II}}$ & 0.6 & 1.7 & 0.3 & 0.97 & 0.81 & 0.8 & 58.67 & - & - & - & - \\
\hline$c_{5}^{\mathrm{II}}$ & 0.8 & 0.4 & 0.9 & 0.81 & 0.43 & 0.83 & 3.67 & 0.81 & 0.41 & 0.90 & 0.67 \\
\hline$c_{6}^{\mathrm{II}}$ & 0.6 & 1.5 & 0.1 & 0.99 & 1.07 & 1.15 & 62.33 & - & - & - & - \\
\hline
\end{tabular}

${ }^{\mathrm{a}}$ Mean sizing error is the average value of the absolute estimation errors for correction coefficients.

were obtained by extensive FE analyses with different sets of correction coefficients randomly assumed using the Latin hypercube sampling technique (Press et al. 1992; Olsson and Sandberg 2002).

\section{Development of Three Dimensional Baseline Model Based on Vibration Tests}

The baseline model of the bridge was developed using the approach described above, which particularly involves: step (1) updating the in-plane structural parameters of the 3D preliminary FE model based on the measured vertical and longitudinal modes and then step (2) identifying the out-of-plane structural parameters of the 3D baseline FE model based on the measured transverse modes.

The natural frequencies for the lower five modes and the first mode shape of the in-plane modes, and the natural frequencies for the lower three modes and the first mode shape of the out-ofplane modes were used in the neural network in steps (1) and (2), respectively. Although more natural frequencies and mode shapes could be included, higher mode shapes were ignored due to noise contamination. The correction coefficients are related with the mass and the stiffness matrices of the super- and substructures, and the abutment spring stiffness as follows:

(1) Step 1 (in-plane motion)

$$
\begin{gathered}
\mathbf{M}=\left\{c_{1}^{\mathrm{I}} A^{\text {super }}, c_{2}^{\mathrm{I}} A^{\text {sub }}\right\} \\
\mathbf{K}=\left\{c_{3}^{\mathrm{I}} I_{Y}^{\text {super }}, c_{4}^{\mathrm{I}} I_{Y}^{\text {sub }}\right\} \\
k=\left\{c_{5}^{\mathrm{I}} k_{l 1}^{\text {abut }}, c_{6}^{\mathrm{I}} k_{r 1}^{\text {abut }}, c_{7}^{\mathrm{I}} k_{l 4}^{\text {abut }}, c_{8}^{\mathrm{I}} k_{r 4}^{\text {abut }}\right\}
\end{gathered}
$$

(2) Step 2 (out-of-plane motion)

$$
\begin{gathered}
\mathbf{K}=\left\{c_{1}^{\mathrm{II}} I_{Z}^{\text {super }}, c_{2}^{\mathrm{II}} I_{Z}^{\text {sub }}\right\} \\
k=\left\{c_{3}^{\mathrm{II}} k_{l 1}^{\text {abut }}, c_{4}^{\mathrm{II}} k_{r 1}^{\text {abut }}, c_{5}^{\mathrm{II}} k_{l 4}^{\text {abut }}, c_{6}^{\mathrm{II}} k_{r 4}^{\text {abut }}\right\}
\end{gathered}
$$

in which $A^{\text {super }}$ and $I^{\text {super }}=$ effective shear area and the moment of inertia of the cross section in superstructure; $A^{\text {sub }}$ and $I^{\text {sub }}$ $=$ those in substructures; $k_{l i}^{\text {abut }}$ and $k_{r i}^{\text {abut }}=$ linear spring stiffness in the longitudinal and rotational directions at abutment $i$; and $c^{\mathrm{I}}$ and $c^{\mathrm{II}}=$ correction coefficients at each step for these parameters. The shear areas of the super- and substructures are common parameters for both in-plane and out-of-plane motions of the bridge. In this study, the shear areas were identified from step (1), since the in-plane motions are more dominant and accurately measured than the out-of-plane motions under traffic loads.

5,000 training patterns numerically obtained by FE analyses were used for the training of the neural network, and 1,000 iterations were performed. An output-input pair in the training patterns is obtained by assuming a set of correction coefficients $c \mathrm{~s}$ with the corresponding frequencies and mode shapes being calculated through FE analysis. The ranges of the correction coefficients in the training process of the neural network were chosen from 0.5 to 1.5 for the super- and substructures, and from 0.1 to 1.9 for the abutment spring stiffness values considering their relatively high uncertainties.

In order to verify the performance of the trained neural network, a set of correction coefficients $c$ s were assumed and the frequencies and mode shapes were calculated through FE analysis. By inputting the calculated mode parameters into the trained neural network, a set of correction coefficients were resulted and

\begin{tabular}{|c|c|c|c|c|c|c|c|c|c|c|c|c|c|}
\hline \multicolumn{8}{|c|}{ In-plane structural parameters } & \multicolumn{6}{|c|}{ Out-of-plane structural parameters } \\
\hline$\overline{c_{1}^{\mathrm{I}}}$ & $c_{2}^{\mathrm{I}}$ & $c_{3}^{\mathrm{I}}$ & $c_{4}^{\mathrm{I}}$ & $c_{5}^{\mathrm{I}}$ & $c_{6}^{\mathrm{I}}$ & $c_{7}^{\mathrm{I}}$ & $c_{8}^{\mathrm{I}}$ & $c_{1}^{\mathrm{II}}$ & $c_{2}^{\mathrm{II}}$ & $c_{3}^{\mathrm{II}}$ & $c_{4}^{\mathrm{II}}$ & $c_{5}^{\mathrm{II}}$ & $c_{6}^{\mathrm{II}}$ \\
\hline 0.93 & 0.99 & 0.96 & 1.00 & 1.25 & $-^{\mathrm{a}}$ & 1.24 & $-^{\mathrm{a}}$ & 1.00 & 0.99 & 0.16 & $-^{\mathrm{a}}$ & 0.67 & $-^{\mathrm{a}}$ \\
\hline
\end{tabular}
compared with the assumed ones. The verification results are shown in Table 4 where the assumed (target) correction coefficients are compared with those identified from the neural net-

Table 5. Neural Network Output Using Measurement Data

${ }^{\mathrm{a} N o t}$ available. 
work. It is observed that structural parameters, except the stiffness of the rotational springs at abutments, are well identified. The lack of correlation between the rotational springs and the modal parameters (as shown in Fig. 10) make it difficult to identify the rotational spring stiffness. Therefore, the stiffness of the rotational springs at the abutments were not updated in the baseline FE model.

After the trained neural network was accepted based on the above testing and verification procedure, the modal parameters measured in the field (shown in Table 1 and Fig. 5) were used as the input in the neural network to identify the structural correction coefficients for the Jamboree Road overcrossing. The results are shown in Table 5, indicating that the structural parameters are corrected by up to $84 \%$ of the original values in the preliminary FE model.

Using these correction coefficients, the preliminary FE model was updated to the baseline model. The modal parameters measured in the field (using only the results by the FDD method) are compared with those computed by the baseline model in Table 1. It was demonstrated that the natural frequencies of the baseline model better agree with the reality than those of the preliminary FE model.

\section{Conclusions}

This paper presents the application of a neural network technique for developing a baseline model of a bridge structure, based on vibration tests. This is an essential step in the methodology currently being established by the writers for long-term structural performance monitoring of bridges. Based on the vibration tests on two instrumented highway bridges, the data analysis and the baseline development, the following observations or conclusions can be made:

1. Sensitivity analysis indicates that it is important to measure vibration on the top of a column in both transverse and longitudinal directions in order to detect seismic damage on the columns.

2. The traffic-induced vibration in the vertical direction is twice intensive than that in the transverse direction. The higher modes and the horizontal modes are more noise contaminated than the lower modes and vertical modes.

3. The natural frequencies of the bridge estimated from the bumping test are lower than those from the braking test, indicating the need to study the effect of excitation level on the vibration characteristics of the bridge.

4. The mode shapes estimated by the FDD method appear more reasonable than those by the PR/RD method.

5. The neural network technique is effective in identifying the structural parameters based on vibration measurement, despite the incomplete measurement of the mode shapes due to the limited sensor number.

6. The lack of correlation between the rotational stiffness of the bridge abutments and the modal parameters makes it difficult to identify these stiffness values.

With the sensor systems permanently installed on the highway bridges and their baselines established in this study, the long-term structural performance of the bridges will be continuously monitored and evaluated in the future. The effects of environmental conditions (e.g., temperature and moisture) and the level of exciting forces on the baseline parameters will be studied.

\section{Acknowledgments}

This research was supported by the California Department of Transportation (Caltrans) and the Korea Science and Engineering
Foundation (KOSEF). The writers would like to thank Caltrans District 12, Tokyo Sokushin Co., FCI Constructors, and Silverado Constructors for their generous support in traffic control and sensor installation.

\section{References}

Aktan, A. E., Catbas, F. N., Grimmelsman, K. A., and Tsikos, C. J. (2000). "Issues in infrastructure health monitoring for management." J. Eng. Mech., 126(7), 711-724.

Asmussen, J. C., and Brincker, R. (1996). "Estimation of frequency response function by random decrement." Proc., 14th Int. Modal Analysis Conf., Society for Experimental Mechanics, Dearborn, Mich., 246-252.

Bendat, J. S., and Piersol, A. G. (1993). Engineering application of correlation and spectral analysis, Wiley, New York.

Brinker, R., Zhang, L., and Andersen, P. (2001). "Modal identification of output-only system using frequency domain decomposition." Smart Mater. Struct., 10(3), 441-455.

Doebling, S. W., Farrar, C. R., and Prime, M. B. (1998). "A summary review of vibration-based damage identification methods." Shock Vib. Dig., 30(2), 91-105.

Ewins, D. J. (1999). Modal testing: Theory, practice and application, Wiley, New York.

Federal Highway Administration (FHWA). (1996). "Seismic design of bridges design example no. 6: Three-span continuous CIP concrete box bridge." Rep. No. FHWA-SA-97-011, Washington, D.C.

Feng, M. Q., and Bahng, E. Y. (1999). "Damage assessment of jacketed RC columns using vibration tests.” J. Struct. Eng., 125(3), 265-271.

Feng, M. Q., De Flaviis, F., and Kim, Y. J. (2002). "Use of microwaves for damage detection of fiber reinforced polymer-wrapped concrete structures." J. Eng. Mech., 128(2), 172-183.

Masri, S. F., Smyth, A. W., Chassiakos, A. G., Caughey, T. K., and Hunter, N. F. (2000). "Application of neural networks for detection of changes in nonlinear systems." J. Eng. Mech., 126(7), 666-676.

Olsson, A. M., and Sandberg, G. E. (2002). "Latin hypercube sampling for stochastic finite element analysis." J. Eng. Mech., 128(1), 121125.

Otte, D., Ponseele, P. V. D., and Leuridan, J. (1990). “Operational shapes estimation as a function of dynamic loads." Proc., 8th Int. Modal Analysis Conf., Society for Experimental Mechanics, Orlando, Fla., 413-421.

Press, W., Teukolsky, S. A., Vetterling, W. T., and Flannery, B. P. (1992). Numerical recipes in $\mathrm{C}$-The art of scientific computing, Cambridge University Press, New York.

Salawu, O. S. (1997). "Detection of structural damage through changes in frequency: a review," Eng. Struct., 19(9), 718-723.

Sanayei, M., McClain, J. A. S., Wadia-Fascetti, S., and Santini, E. M. (1999). "Parameter estimation incorporating modal data and boundary conditions." J. Struct. Eng., 125(9), 1048-1055.

SAP2000 user's manual-nonlinear version 7.1. (1999). Computers and Structures, Inc., Berkeley, Calif.

Yang, J. C. S., Chen, J., and Dagalakis, N. G. (1985). "Damage detection in offshore structures by the random decremental technique." J. Energy Resour. Technol., 106, 38-42.

Yun, C. B., Lee, J. J., Lee, J. W., Yi, J. H., and Jung, H. Y. (2002). "Ambient vibration tests for structural integrity assessment of bridges." Proc., 1st Int. Conf. on Bridge Maintenance, Safety and Management, International Association for Bridge Maintenance and Safety, Barcelona, Spain.

Yun, C. B., Yi, J. H., and Bahng, E. Y. (2001). "Joint damage assessment of framed structures using a neural networks technique." Eng. Struct., 23(5), 425-435. 
Copyright of Journal of Engineering Mechanics is the property of American Society of Civil Engineers and its content may not be copied or emailed to multiple sites or posted to a listserv without the copyright holder's express written permission. However, users may print, download, or email articles for individual use. 